\title{
Structure of Mycobacterium tuberculosis tryptophan synthase: a model system for allosteric inhibition
}

\author{
Karolina Michalska ${ }^{1}$, Samantha Wellington ${ }^{2,3,4}$, Partha P. Nag ${ }^{2}$, Robert \\ Jedrzejczak ${ }^{1}$, Natalia I. Maltseva ${ }^{1}$, Stewart L. Fisher ${ }^{2}$, Stuart L. Schreiber ${ }^{2,6}$, \\ Deborah T. Hung ${ }^{1,2,3}$ Andrzej Joachimiak ${ }^{1}$
}

\author{
${ }^{1}$ Center for Structural Genomics of Infectious Diseases, University of Chicago, Chicago, USA \\ ${ }^{2}$ Broad Institute or MIT and Harvard, Cambridge, USA \\ ${ }^{3}$ Department of Microbiology and Immunobiology, Harvard Medical School, Boston, USA \\ ${ }^{4}$ Department of Molecular Biology and Center for Computational and Integrative Biology, \\ Massachusetts General Hospital, Boston, USA \\ ${ }^{5}$ Harvard School of Public Health, Boston, USA \\ ${ }^{6}$ Department of Chemistry and Chemical Biology, Harvard University, Cambridge, USA
}

The survival of many bacteria, including pathogenic species, depends on the ability to synthesize the amino acid L-tryptophan whenever it is not available from the environment. The pathway for L-Trp biosynthesis is typically organized in a single strictly regulated $\operatorname{trp}$ operon and most often include $\operatorname{trp} E, \operatorname{trp} G, \operatorname{trp} D, \operatorname{trp} C, \operatorname{trp} B$ and $\operatorname{trp} A$ genes. The $\operatorname{trp} A$ and $\operatorname{trp} B$ genes encode $\alpha$ and $\beta$ subunits of a tetrameric $\alpha_{2} \beta_{2}$ tryptophan synthase $(\operatorname{Trp} A B)$, catalyzing the last two reactions of L-Trp biosynthesis. The tryptophan biosynthetic pathway was validated as conditionally essential for the survival of Mycobacterium tuberculosis (Mtb) during infection. This pathway is absent in humans and has a high potential for anti-tuberculosis drug targets. We have expressed, purified and crystallized active $\alpha_{2} \beta_{2} \operatorname{Trp} A B$ from Mtb. A novel ligand was identified at the Broad Institute using library screening as a growth inhibitor of Mtb and targeting TrpA. We have determined several crystal structures of the enzyme in apo-form and in complex with a new inhibitor. The results of structural studies combined with functional characterization shows that the ligand is an allosteric inhibitor and binding in a highly unique fashion at the interface between TrpA and TrpB. Unexpectedly, though the compound potently inhibits the overall production of tryptophan, it increases the enzyme's affinity for substrates and stabilizes closed, active conformation of TrpB. In contrast to substrate mimetics, it affects multiple steps of the TrpAB reaction, drastically slowing the rate of both the $\operatorname{TrpA}$ and $\operatorname{TrpB}$ reactions. The new ligand represents a useful probe for understanding Mtb TrpAB regulation, function, and catalytic mechanism. Our findings suggest that allosteric inhibition can be a powerful strategy for targeting metabolic enzymes, and support the effectiveness of such strategies targeting functions that are required for in vivo infection, despite their apparent dispensability in the presence of tryptophan under in vitro conditions.

CSGID is funded by the NIAID of NIH (contract No.: HHSN272200700058C and HHSN272201200026C) and Gates Foundation though SDDC. The use of SBC beamlines at the Advanced Photon Source was supported by the U.S. Department of Energy, OBER (contract DE-AC02-06CH11357). 